\title{
Da tradição a técnica: perspectivas e realidades da agricultura de derruba e queima
}

\section{na Amazônia}

\author{
From tradition to technique: perspectives and realities of felling and burning agriculture in
}

Amazon

De la tradición a la técnica: perspectivas y realidades de la agricultura de tala y quema en la Amazonía

\author{
Alasse Oliveira da Silva \\ ORCID: https://orcid.org/0000-0003-2230-1747 \\ Universidade Federal Rural da Amazônia, Brasil \\ E-mail: alasse.oliveira77@gmail.com \\ Aline Oliveira da Silva \\ ORCID: https://orcid.org/0000-0002-9575-271X \\ Universidade Federal Rural da Amazônia, Brasil \\ E-mail: oliveiraaline141@gmail.com \\ Dayla Carolina Rodrigues Santos \\ ORCID: https://orcid.org/0000-0002-6227-626X \\ Universidade Federal Rural da Amazônia, Brasil \\ E-mail: daylas70@gmail.com \\ Isabelle Caroline Bailosa do Rosário \\ ORCID: https://orcid.org/0000-0002-2572-7973 \\ Universidade Federal Rural da Amazônia, Brasil \\ E-mail: carolinebailosa@gmail.com \\ Henrique da Silva Barata \\ ORCID: https://orcid.org/0000-0001-6356-4629 \\ Universidade Federal Rural da Amazônia, Belém \\ E-mail: henriquebarata2000@gmail.com \\ Lucas Lima Raiol \\ ORCID: https://orcid.org/0000-0003-4080-9333 \\ Universidade Federal Rural da Amazônia, Brasil \\ E-mail: lucasraiolsk8@gmail.com
}

\begin{abstract}
Resumo
O sistema de derruba e queima ainda é praticado na Amazônia, contribuindo para a destruição ambiental. Objetivouse apresentar as perspectivas e realidades da agricultura de corte e queima na Amazônia, bem como suas relações envolvidas. Utilizou-se um levantamento bibliográfico para realização do estudo. Os agricultores persistem nesse sistema, por ser de baixo custo e de fácil adoção, em função da baixa fertilidade da maioria dos solos da região amazônica, do elevado custo de fertilizantes e corretivos. Os principais focos atuais de queimadas na Amazônia são associados à agricultura, em especial em escala familiar. As queimadas praticadas proporcionam danos ao ambiente, fauna e flora. Esse sistema mostra-se insustentável ainda presente no ambiente agrícola, principalmente na agricultura familiar da região Norte do Brasil, fator este agravante para a fauna e a flora local.
\end{abstract}

Palavras-chave: Queimadas; Agricultura; Amazônia; Agricultura familiar.

\begin{abstract}
The felling and burning system is still practiced in the Amazon, contributing to environmental destruction. The objective was to present the perspectives and realities of slash and burn agriculture in the Amazon, as well as their relations involved. A bibliographic survey was used to carry out the study. Farmers persist in this system, as it is low cost and easy to adopt, due to the low fertility of most soils in the Amazon region, the high cost of fertilizers and correctives. The main current focuses of burning in the Amazon are associated with agriculture, especially on a family scale. The fires practiced cause damage to the environment, fauna and flora. This system, proves to be unsustainable still present in the agricultural environment, mainly in family farming in the Northern region of Brazil, an aggravating factor for the local fauna and flora.
\end{abstract}

Keywords: Burnings; Agriculture; Amazon; Family farming. 


\section{Resumen}

El sistema de tala y quema todavía se practica en la Amazonía, lo que contribuye a la destrucción ambiental. El objetivo fue presentar las perspectivas y realidades de la agricultura de roza y quema en la Amazonía, así como las relaciones involucradas. Para la realización del estudio se utilizó una encuesta bibliográfica. Los agricultores persisten en este sistema, ya que es de bajo costo y fácil de adoptar, debido a la baja fertilidad de la mayoría de los suelos de la región amazónica, el alto costo de fertilizantes y correctivos. Los principales focos actuales de quema en la Amazonía están asociados a la agricultura, especialmente a escala familiar. Los incendios practicados provocan daños al medio ambiente, fauna y flora. Este sistema, resulta insostenible aún presente en el medio agrícola, principalmente en la agricultura familiar en la región norte de Brasil, un agravante para la fauna y flora local.

Palabras clave: Quemaduras; Agricultura; Amazonia; Agricultura familiar.

\section{Introdução}

Nos anos de 1960 e 1970, os Planos de Desenvolvimento da Amazônia (PDA) foram orientados para contribuir com a inserção de grandes projetos agrícolas e pecuários (Fearnside, 1997). Nesse período, projetos de colonização foram criados, para que pudesse integrar economicamente a região, atraindo milhares de famílias de produtores rurais (Santos, 2008). Dessa maneira, houve uma aceleração do desmatamento no território (MMA, 2000).

Na Amazônia, o principal uso da terra é relacionado à agricultura de subsistência, sendo praticada por produtores em pequena escala, por meio do corte e queima da floresta primária ou secundária, ou seja, a floresta nativa é retirada pelo método de derruba e queima, a cultura é instalada e cultivada até que a fertilidade do solo decresça ao nível de degradação. Posteriormente, os agricultores realizam um período de pousio, permitindo que a vegetação secundária se desenvolva, a qual, em seguida é cortada e queimada, começando um novo ciclo (Freitas et al., 2013; Santos, 2008).

Desse modo, o princípio básico dessa prática é a utilização da biomassa florestal queimada e revolvida no solo como uma fonte de nutrientes para cultivos agropecuários (Santos, 2008). Entretanto, esse método ocasiona diversos problemas ambientais, sociais e econômicos, especialmente, em lugares muito habitados e com restrição de terrenos destinados a expansão agrícola (Freitas et al., 2013). Apesar disso, é provável que os produtores permaneçam utilizando os sistemas de derruba e queima devido à dificuldade de acessar os recursos que permitiriam a escolha por sistemas sustentáveis, com um melhor rendimento e maior duração (Santos, 2008).

Nesse contexto, os agricultores são afetados com a redução da produtividade agrícola, e geralmente não possuem recursos financeiros para o restabelecimento da fertilidade do solo, por isso, deslocam-se para novas áreas (Rego; Kato, 2017). Assim, o método da agricultura migratória, pode ocasionar degradação direta no ecossistema, devido à retirada da biomassa $\mathrm{e}$ perda de nutrientes através da lixiviação e volatilização, ocasionando a baixa produção, gerando ciclos de pobreza e abandono do terreno utilizado (Freitas et al., 2013).

Dessa maneira, devido à diminuição da capacidade produtiva do solo, ocasionada por essa prática, é fundamental a utilização de novos sistemas, nos quais, a produção é realizada de forma sustentável e variada (Rego \& Kato, 2017). Por exemplo, os sistemas agroflorestais possuem um alto grau de biodiversidade, apresentando estruturação horizontal e vertical mais próxima à estrutura de uma floresta nativa (Nair, 1993). Por isso, a recomendação de Sistema agroflorestal (SAF) para pequenos agricultores é analisada como uma alternativa para otimizar o uso das áreas alteradas e abandonadas, haja vista, o melhor aproveitamento para cultivos agrícolas e florestais, além disso, protege e melhora as propriedades químicas, biológicas e físicas do solo (Freitas, 2008).

Diante disso, este trabalho teve como objetivo apresentar as perspectivas e realidades da agricultura de corte e queima na Amazônia, bem como entender seus aspectos motivadores, o ambiente em questão e as alternativas sustentáveis atuais através de um levantamento bibliográfico. 


\section{Metodologia}

Para obtenção das informações pertinentes ao objetivo da pesquisa, foi utilizado o método de revisão bibliográfica, realizando um compilado sobre as principais abordagens teóricas metodológicas publicadas, com destaque para as discussões nos contextos da agricultura familiar e transição agroecológica.

A pesquisa bibliográfica foi realizada através dos principais meios de busca de bases de dados eletrônicos científicos, relacionados agricultura de corte e queima na Amazônia. A busca dos dados ocorreu durante o segundo semestre de 2019 até o início de 2020, com a inclusão e identificação de fontes bibliográficas sobre o assunto.

As bases de dados utilizadas foram o periódico CAPES/MEC, Scientific Eletronic Library Online-Scielo e Google Acadêmico.

As buscas das palavras chaves sobre o tema foram realizadas em dois idiomas, em português e inglês. Os termos utilizados juntos ou mesmo separados, nas respectivas bases de dados foram:

- Sistema derruba e queima na Amazônia/ System drops and burns in the Amazon

- $\quad$ Agricultura de derruba e queima/ Felling and burning agriculture

- Queima da capoira na Amazônia/ Capoeira burning in the Amazon

- $\quad$ Sistema tipitamba/ Tipitamba system

- Transição agroecológica na Amazônia/ Agroecological transition in the Amazon

- $\quad$ Agricultura de corte e queima na Amazônia/ Slash and burn agriculture in the Amazon

- $\quad$ O ambiente, a fauna e a flora/ The environment, fauna and flora

- Agricultura Familiar e o sistema de derruba e queima/ Family farming and the tipping and burning system

- $\quad$ Alternativas sustentáveis para a Amazônia/ Sustainable alternatives for the Amazon

Após a coleta das informações, selecionaram-se os que atendiam o objetivo do estudo, não se estabelecendo limite de ano para o material uma vez que as informações presentes na literatura sobre o assunto são escassas.

\section{Resultados e Discussão}

\section{a. Agricultura de derruba e queima na Amazônia: aspectos motivadores}

A cultura do derruba e queima na Amazônia é uma das principais práticas de cultivo, conhecida também como agricultura itinerante ou coivara, este sistema consiste na derruba da vegetação primária e rotação de culturas anuais (mandioca, milho, feijão) com a vegetação secundária, denominada de capoeira (Kato et al., 2007).

Em torno de 75\% da região Bragantina no nordeste do Estado do Pará é coberto de vegetação secundária no lugar da floresta primária decorrente da prática derruba e queima (Ferreira \& Oliveira, 2001). E de acordo com Oliveira et al. (2007), este sistema produtivo é o que prevalece na região amazônica, principalmente na agricultura familiar e de subsistência, para formação de roças e geração de alimento devido sua praticidade e benefícios trazidos a curto prazo.

Nesse sistema de coivara, o produtor familiar realiza a queima no final do período de pousio, entre dois períodos de cultivo, quando a vegetação secundária conhecida como capoeira, cresce após o período de cultivo é cortada, seca e queimada, para disponibilizar matéria orgânica ao solo, como fertilizante e corretivo de acidez, as cinzas resultantes da queimada, contêm nutrientes acumulados pela vegetação secundária (Sá et al., 2007). Essa prática busca suprir as necessidades nutricionais das culturas com os nutrientes acumulados na vegetação secundária (Marcolan, Locatelli \& Fernandes, 2009).

Os agricultores persistem nesse sistema, por ser de baixo custo e de fácil adoção, em função da baixa fertilidade da maioria dos solos da região amazônica, do elevado custo de fertilizantes e corretivos. Outro fator é decorrente da insuficiência de políticas adequadas de fomento e assistência técnica levando os produtores a fazer uso da prática derruba e queima pelo fato 
de muitas vezes não haver a troca de conhecimento entre um profissional da área agrária com os agricultores familiares, isso acarreta a carência de técnicas e insumos que possibilite o melhor aproveitamento da terra sem provocar grandes degradações, evitando o uso do sistema de coivara (Sá et al., 2007).

No entanto, vale ressaltar que os benefícios da prática do derruba e queima são temporários, a longo prazo (sete anos) o processo de queima da vegetação possui diversas desvantagens como a perda dos nutrientes retidos na biomassa, tais como, as perdas de até $96 \%$ de nitrogênio, $76 \%$ de enxofre, $47 \%$ de fósforo, $48 \%$ de potássio, $35 \%$ de cálcio e $40 \%$ de magnésio, o que resulta na degradação do solo e no declínio da produtividade (Mackensen et al., 1996; Marcolan, Locatelli \& Fernandes, 2009).

Mudar esse sistema do derruba e queima na Amazônia é um dos principais desafios para a agricultura brasileira, não só pelos aspectos tecnológicos e econômicos, mas pela tradição milenar do uso dessa tecnologia repassado de geração em geração, pelo fato de estar intrinsecamente enraizado na tradição regional desde a existência de uma agricultura précolombiana e indígena (Alves et al., 2020).

Dessa maneira, faz-se necessário a mudança de prática, uma vez que a agricultura de corte e queima se torna insustentável na medida em que são feitas repetidas queimadas, o que diminui o tempo de pousio entre os cultivos. E para esse sistema ser sustentável em termos de produção, o pousio necessita variar de sete a dez anos, o que normalmente não ocorre (Denich, Kanashiro \& Vlek, 1999).

\section{b. Agricultura Familiar e o sistema de derruba e queima}

A agricultura familiar atualmente é uma das principais responsáveis pelo avanço do desmatamento da região amazônica por meio do sistema de derruba e queima, esse sistema é altamente prejudicial em diversas escalas, seja ela para a atmosfera pela liberação de gás carbônico, contribuindo para o aumento do efeito estufa, perda de vegetação nativa, prejudicial ao solo pelo fato de haver a retirada da cobertura verde e pela lixiviação de nutrientes por lâmina de água e por evaporação das cinzas advindas da biomassa vegetal (Sá, 2007).

Dessa forma, é possível fazer uma reflexão sobre os impactos em pequena, média e grande escala no campo. Tal problemática se dá por não haver o manejo correto do solo, além de se agravar pelo fato de não apenas retirar a vegetação mas também fazer a queima dela. Os principais focos atuais de queimadas na Amazônia são associados à agricultura, em especial em escala familiar (Sá, 2007).

A falta de conhecimento técnico é um dos fatores que possibilita que os agricultores familiares continuem fazendo o uso do sistema derruba e queima, sem a implementação de alternativas mais sustentáveis na exploração do solo e de seus recursos. O conhecimento tradicional passado de geração em geração sobre a coivara permanece enraizado no cenário da agricultura familiar até os dias atuais, dificultando a aceitação de novas práticas e sistemas capazes de trazer mais sustentabilidade no cenário agrário (Ferreira \& Oliveira, 2001).

A assistência técnica é uma forma de fomentar a aceitação e implantação de novos sistemas nas propriedades, é também uma chave importante para a conscientização e fiscalização para diminuição do desmatamento e perda de recursos naturais. Uma problemática recorrente do sistema itinerante é a derrubada da floresta secundária que vem a trazer prejuízos que poderiam ser evitados com o acompanhamento de técnicos capacitados para fazer a orientação no uso da área, da vegetação local que é de suma importância. Em muitas situações, essa vegetação é a única fonte desses para sustentabilidade da agricultura familiar (Ferreira \& Oliveira, 2001).

Segundo Kato et al. (2004a), a agricultura familiar na Amazônia Oriental, particularmente na região nordeste do Pará, caracteriza-se pela prática da agricultura rotacional. Porém, para desenvolver essa agricultura o pequeno produtor passa por diversas dificuldades. 
Dentre elas estão, a questão econômica, sendo um fator limitante para que o agricultor familiar implemente novas técnicas na produção, os insumos geralmente apresentam custos elevados, consequentemente leva o agricultor familiar a fazer o uso sistema de derruba e queima, pois é uma forma mais simplificada e sem grandes gastos pelo fato de não usarem maquinário mais avançado na propriedade, porém é uma prática altamente desfavorável para o meio ambiente (Kato et al. 2004a).

Na região do nordeste paraense o uso do sistema derruba e queima na maioria das vezes são em propriedades cujo sistema de produção está em torno do plantio de culturas como o feijão caupi (Vigna unguiculata), mandioca (Manihot esculenta) e milho (Zea mays) que necessitam de um solo rico em nutrientes (Cravo et al. ,2006).

Para diminuir impactos ambientais no solo e na vegetação, advindos do mal uso do solo e de outros recursos naturais, desenvolveu-se um novo sistema cuja função é fazer uso da biomassa da vegetação derrubada da capoeira, devolvendo os nutrientes contidos na composição das plantas da região, sem fazer uso do fogo (Kato et al., 2003).

O sistema de corte e trituração como é chamado, constitui uma alternativa para a agricultura de corte e queima, sem alterar o sistema de produção predominante na região Amazônica sendo uma alternativa mais sustentável ambientalmente pois em vez de queimar, a vegetação retirada da capoeira é reintegrada ao solo para devolver a fertilidade do mesmo (Kato et al. 2003).

Uma das funções das capoeiras, que vem sendo utilizada como suporte à maioria das experiências de produção agrícola familiar na Amazônia, é a associada ao suprimento de nutrientes e de matéria orgânica (Kato et al., 2004).

\section{c. O ambiente, a fauna e a flora}

De acordo com Kato et al. (2007), o sistema de derruba e queima é o processo mais executado por aqueles que praticam a agricultura familiar, uma vez que o conhecimento tradicional adquirido de forma hereditária dá lugar à insustentabilidade do campo, com a degradação do solo, perda de nutrientes e da microfauna do solo.

Os autores corroboram que, este tipo de atividade de pré-plantio, gera impactos negativos muito mais severos ao meio ambiente, seja de forma direta ou indiretamente, tais ações corroboram para o desgaste progressivo do ecossistema a qual está inserido.

Segundo Silva e Silva (2015), a diminuição do potencial biológico da terra no sistema de derruba e queima, pode levar a processos de desertificação causando alterações em níveis biogeofisicos, biogeoquímicos e sociais tanto em escala temporal como espacial, acrescentam ainda que a quebra do equilíbrio natural ocorre devido à sobrecarga suporte físico do ecossistema, que por consequência reduz a biodiversidade local, comprometendo também a fauna e flora e nascentes de rios.

Estudos de hidrogeoquímicas com foco em escoamento superficial da água em diversos sistemas de cultivo realizado por Costa (2011; 2013), demonstraram que as áreas que mais tiveram perdas significativas de nutrientes como cálcio, magnésio, potássio e sulfato foram os sistemas de derruba e queima.

O que pode ser justificado por Thomaz (2013), que destaca os processos erosivos e sua relação com o escoamento superficial, quanto maior for à densidade, mais é afetada a estabilidade dos agregados do solo, reduzindo o teor de nutrientes pela lixiviação e volatização, além da redução do carbono.

De acordo com Silva et al. (2011) a liberação do CO2 produzido durante a queima traz impactos diretos no ciclo biogeoquímico do nitrogênio e carbono, além da perda de nutrientes para a atmosfera no processo de volatilização, mas também pode ocorrer pelo arraste de partículas geralmente a movimentos ascendentes.

Segundo dados do Instituto Brasileiro do Meio Ambiente e dos Recursos Naturais Renováveis- IBAMA (2013), as principais causas de queimadas e incêndios na vegetação foram em decorrência de renovação de pastagens com participação de $31 \%$, para fins de rebrota do pasto e queima no preparo de solo com $22 \%$ como método de limpeza de área. 
Vale ressaltar que tal prática por ser realizada por indivíduos de baixa escolaridade não possuem conhecimento da relação causa e efeito, não apenas para o meio ambiente e clima, mas também para a própria saúde do agricultor e dos animais que habitam o local, pois baseiam-se apenas no saber tradicional adquirido da experimentação diária no campo, que por consequência faz parte da construção da identidade social, carregando muitas vezes o "status" de cultura do fogo (Dias, 2012).

Todavia, Sollins et al. (1984), abordam em suas pesquisas os benefícios do sistema corte e queima, principalmente relacionado a decomposição rápida de matéria orgânica e as cinzas resultantes do processo, que rapidamente é transportada para o perfil do solo por meio das chuvas, isso ocorre devido à alta mobilidade dos nutrientes que tornam-se disponíveis na solução do solo.

Os autores acreditam que, este mecanismo é mais indicado para o curto prazo, como por exemplo as pastagens, quando a técnica se estende para outros ciclos de plantio com menor tempo de reocupação ou (pousio), o mesmo tende a decair a produção devido o esgotamento do solo e a redução da biomassa da capoeira.

Outro aspecto que pode ser abordado como benefício desta prática é o controle direto das comunidades de artrópodes, que são consideradas na agricultura tradicional como pragas da lavoura, contudo o fogo não elimina apenas as pragas, mas também seus inimigos naturais que coexistem no ecossistema (Santos \& Alegre, 2007).

Ainda de acordo com os autores, algumas espécies de formigas predam as larvas de lepidópteras e coleópteras, que podem causar secamento, cloroses, baixo desenvolvimento do planeta entre outros sintomas, além de causar perdas na produtividade podendo chegar até $50 \%$ de dano econômico a depender o tipo de inseto.

As formigas são forte aliadas no controle biológico de insetos, sendo consideradas como indicadores de qualidade de manejo, principalmente em capoeiras ou floretas secundárias em regeneração, sendo favorecidas por áreas onde a biomassa e a composição de nutrientes esteja em equilíbrio, ou seja, áreas que não tenham passado pela queimada, afim de atrair e estabelecer as espécies da macrofauna, os autores reiteram que quanto maior for o equilíbrio ecológico mais diverso será a população de formigas e mais eficaz o controle de pragas (Santos \& Alegre, 2007).

A queima também traz perdas significativas na flora, plantas que provavelmente não chegaram a ser exploradas, e que possuem atrativo econômico e potencial terapêutico, como as plantas medicinais que ocorrem nas capoeiras, que acabam sendo perdidas pelo fogo, ou as sementes de espécies nativas que perdem seu vigor germinativo pelas altas temperaturas a qual o solo é submetido, potenciais estes que poderiam agregar a renda dos agricultores (Melo \& Durigan, 2010).

\section{d. Alternativas sustentáveis atuais}

\section{Transição agroecológica}

É necessária uma nova ótica para a produção agrícola no Brasil, sobretudo na Amazônia que cada vez mais sofre pressões sobre a floresta e uso do solo. Nesse sentido a pesquisa aborda estratégias para a construção de bases ecológicas e sustentáveis. Com base nisso uma das alternativas é a transição agroecológica (Sousa, Araújo \& Wandelli, 2015).

A agroecologia surge como uma alternativa de práticas e conhecimentos, a mesma propõe um conjunto de princípios (socioeconômicos, agronômicos, valores culturais, éticos e tradicionais) e metodologias participativas que dão suporte ao processo de transição dos modelos de agricultura convencional para o de agricultura de desenvolvimento rural sustentável (Sousa, Araújo \& Wandelli, 2015).

Segundo Gliessman (2000) a transição agroecológica passa por etapas internas e externas do sistema de produção, a depender da distância da sustentabilidade. As condições internas são destacadas a eliminação do uso do fogo, diminuição e racionalização de insumos químicos, substituição de insumos químicos por orgânicos, adaptações dos sistemas produtivos e manejo da biodiversidade, e em relação à transição externa do sistema, a transição agroecológica não pode ser exclusivamente fundamentada no uso de tecnologias, ainda que as mesmas sejam essenciais, porém a transição agroecológica depende de 
outros fatores externos inerentes a unidades de produção estabelecidos, podendo assim ser alcançado (Gliessman, 2001; Mattos et al., 2010).

Dessa forma, há uma construção de condições da sociedade e do estado que fortalecem a transição agroecológica, sob suas particularidades e perspectiva do desenvolvimento de uma consciência pública; da organização dos mercados consumidores; das mudanças na pesquisa, ensino e extensão; da disponibilização de infraestrutura de transporte, energia elétrica, comunicação, educação e saúde; da formulação de politicas públicas com enfoque agroecológico; inovações na legislação ambiental e politicas de crédito rural no contexto agroecológico (Mattos et al., 2010).

\section{Sistema Tipitamba}

A necessidade da busca de práticas que substituem o corte e a queima da capoeira para o preparo das áreas agrícolas levou a iniciativas de pesquisas como o projeto SHIFT - Capoeira, conhecido atualmente como Tipitamba, desenvolvido pela Embrapa e por universidades alemães (Sampaio, Kato, Nascimento \& Silva, 2008).

O projeto Tipitamba compõe o Programa Nacional de Sistema de Produção Florestal e Agroflorestal da Embrapa, sendo inserido no projeto "Aperfeiçoamento e validação de tecnologias de manejo de capoeiras que visa ao uso sustentado da capoeira como vegetação de pousio na agricultura da Amazônia Oriental", tratando-se, portanto de uma iniciativa conjunta que integra mais cinco subprojetos: aperfeiçoamento e teste de equipamento visando técnicas de preparo de área sem o uso do fogo, melhoramento das capoeiras com árvores leguminosas de rápido crescimento, integração da pecuária bovina no ciclo da capoeira e avaliação socioeconômica e valoração da tecnologia sem queima e capoeira melhorada (Sampaio, Kato, Nascimento \& Silva, 2008).

O sistema Tipitamba contribui para a melhora da produtividade do solo, melhor uso da terra, preservação do meio ambiente e toda biomassa aérea da vegetação secundária no sistema de corte e trituração é fonte de matéria orgânica para o sistema, contribuindo para um balanço positivo de nutrientes (Sampaio, Kato, Nascimento \& Silva, 2008).

\section{Sistemas Agroflorestais (SAF's)}

Os sistemas agroflorestais é caracterizado pelo cultivo de espécies perenes (lenhosas) juntamente com espécies herbáceas (cultivos anuais e/ou pastagens), e suas interações ecológicas favorecem a ciclagem de nutrientes, otimização do uso da energia solar, manutenção da umidade do solo e pela proteção do solo contra a erosão e a lixiviação, além também de benefícios econômicos, ou seja, os SAF's contribuem para um sistema produtivo e sustentável (Alves, 2009).

A definição dos SAF's é resultante de diversas práticas, discussões e pesquisas, na qual inclui os processos de sucessão ecológica e conceitos de sustentabilidade e agroecologia, com isso existe dois principais modelos (Tabela 1) de SAF's aplicados para diversas finalidades, sendo as linhas: convencional e agroecológica (Alves, 2009).

Tabela 1 - Principais diferenças das duas linhas de SAF's.

\begin{tabular}{ll}
\hline \multicolumn{1}{c}{ Agroecológica } & \multicolumn{1}{c}{ Convencional } \\
\hline Plantio adensado das árvores & Plantio no espaçamento final \\
Maior estabilidade ecológica e econômica, rápido & Menor acúmulo de matéria orgânica (biomassa) \\
acúmulo de matéria orgânica & \\
$\begin{array}{l}\text { Maior flexibilidade para efetuar mudanças no } \\
\text { sistema, conforme demandas de mercado e }\end{array}$ & $\begin{array}{l}\text { O produtor fica preso a um sistema rígido, com a } \\
\text { trajetória pré-determinada e poucas possibilidades de } \\
\text { disponibilidade de mão de obra, etc. }\end{array}$ \\
\hline
\end{tabular}


Segundo Carvalho (2008), a produção dos SAF's na Amazônia vêm se tornando cada vez mais comum em organizações de pequenos produtores de base familiar, em associações ou cooperativas, geralmente localizadas em áreas de difícil acesso e pouca infraestrutura.

Pode-se citar como exemplo o município de Tomé-Açú, na qual apresenta diversas associações e cooperativas de produtores rurais, com destaque para a Cooperativa Agrícola Mista de Tomé-Açu (CAMTA) e a Associação de Produtores e Produtoras de Agricultura Familiar do Município de Tomé-Açu (APPRAFAMTA), sendo a CAMTA como grande protagonista no desenvolvimento do município através da agroindustrialização da produção agrícola, e prestação de assistência técnica aos agricultores cooperados e não cooperados (Rego \& Kato, 2017).

\section{Considerações Finais}

A agricultura de derruba e queima na Amazônia mostra-se como um sistema insustentável ainda presente no ambiente agrícola, principalmente na agricultura familiar da região Norte do Brasil, fator este agravante para a fauna e a flora local.

Os estudos demostram os efeitos negativos que o sistema de derruba e queima ocasiona ao meio ambiente e as gerações futuras, portanto, espera-se que nos próximos anos ocorra, mesmo que de forma gradativamente, uma substituição dessa técnica, caso existiam políticas públicas eficientes e abrangentes, assistência técnica e extensão rural que busquem promover e incentivar a utilização de práticas sustentáveis na agricultura amazônica.

Além disso, as alternativas sustentáveis atuais (transição agroecológica, técnica do sistema tipitamba e os sistemas agroflorestais) configuram-se como sendo importantes práticas que podem ser adotadas pelos agricultores dessa região.

\section{Referências}

Alves, L. M. (2009). Sistemas Agroflorestais (SAF's) na restauração de ambientes degradados. Programa de Pós-graduação em Ecologia Aplicada ao Manejo e Conservação de Recursos Naturais, UFJF, Juiz de Fora.

Alves, R., \& Modesto Junior, M. D. S. (2020). Roça sem fogo: da tradição das queimadas à agricultura sustentável na Amazônia. Embrapa Amazônia Oriental-Livro técnico (INFOTECA-E). http://www.infoteca.cnptia.embrapa.br/infoteca/handle/doc/1119432

Carvalho, O. M. D., Neto, J. M. D. S., Carvalho, E. M. D., \& Pereira, S. A. (2008). A Nova Economia Institucional-N Ei E Os Sistemas Agroflorestais-Saf'S: Um Estudo Com Duas Organizações Agroflorestais Na Região Amazônica (No. 1349-2016-107258).

Costa, C. D., Figueiredo, R. D. O., \& Santos, I. D. O. (2011). Influência do uso da terra no Nordeste Paraense sobre indicadores físico-químicos de qualidade da água do escoamento superficial no solo. In Embrapa Meio Ambiente-Artigo em anais de congresso (ALICE). In: SIMPÓSIO BRASILEIRO DE RECURSOS HÍDRICOS, 19, 2011, Maceió. Anais... Maceió: Associação Brasileira de Recursos Hídricos, 2011. 15p..

Costa, C.F.G. (2011). Hidrogeoquímica do escoamento superficial em solos de uma mesobacia no nordeste paraense. (Dissertação Mestrado em Ciências Florestais). Universidade Federal Rural da Amazônia, Belém. http://ainfo.cnptia.embrapa.br/digital/bitstream/item/56718/1/2011TS09.pdf

da Costa, C. F., Figueiredo, R. D. O., Oliveira, F. D. A., \& Santos, I. P. D. O. (2013). Escoamento superficial em Latossolo Amarelo distrófico típico sob diferentes agroecossistemas no nordeste paraense. Revista Brasileira de Engenharia Agrícola e Ambiental, 17(2), 162-169. 10.1590/S141543662013000200007

de Oliveira Silva, A. K., \& de Barros Silva, H. P. (2015). O processo de desertificação e seus impactos sobre os recursos naturais e sociais no município de Cabrobó-Pernambuco-Brasil. PRACS: Revista Eletrônica de Humanidades do Curso de Ciências Sociais da UNIFAP, 8(1), 203-215. DOI: http://dx.doi.org.10.18468/pracs.

Denich, M., Kanashiro, M., \& Vlek, P. L. G. (1999). The potential and dynamics of carbon sequestration in traditional and modified fallow systems of the Eastern Amazon region, Brazil (pp. 213-229). CRC Press: Boca Raton, FL, USA. 10.1201/9780203753187-11.

Dias, G. F. (2009). Queimadas e incêndios florestais cenários e desafios: subsídio para a educação ambiental. Brasília: IBAMA, 2.

Fearnside, P. M. (1997). Limiting factors for development of agriculture and ranching in Brazilian Amazonia. 57(40):531-549.

Ferreira, M., \& de Oliveira, L. C. (2001). Potencial produtivo e implicações para o manejo de capoeiras em áreas de agricultura tradicional no nordeste paraense. Embrapa Amazônia Oriental-Comunicado Técnico (INFOTECA-E). http://www.infoteca.cnptia.embrapa.br/infoteca/handle/doc/403377

Freitas, J. D. L. (2008). Sistemas agroflorestais e sua utilização como instrumento de uso da terra: o caso dos pequenos agricultores da ilha de Santana, Amapá, Brasil. 2008. 247f (Tese de doutorado). Universidade Federal Rural da Amazônia, Belém. 
da Luz Freitas, J., dos Santos, E. S., Lima, R. B., \& de Lima Silva, T. (2013). Comparação e análise de sistemas de uso da terra de agricultores familiares na Amazônia. Biota Amazônia (Biote Amazonie, Biota Amazonia, Amazonian Biota), 3(1), 100-108. http://dx.doi.org/10.18561/21795746/biotaamazonia.v3n1p100-108.

Gliessman, S. R. (2001). Agroecologia: processos ecológicos em agricultura sustentável. Ed. da Univ. Federal do Rio Grande do Sul, UFRGS. 653.

EM, I. A. Instituto Brasileiro do Meio Ambiente e dos Recursos Naturais Renováveis IBAMA. https://www.mma.gov.br/component/k2/item/11586-ibamainstituto-brasileiro-do-meio-ambiente-e-dos-recursos-naturais-renovaveis

Kato, O. R., Kato, M. D. S. A., Vielhauer, K., Block, A., \& Jesus, C. D. (2003). Cultivo do milho em sistema de corte e trituração da capoeira na região nordeste do Pará-efeito da época do preparo de área. Embrapa Amazônia Oriental.

Kato, O., Kato, M. D. S., Sá, T. D. A., \& Figueiredo, R. (2004). Plantio direto na capoeira. Embrapa Amazônia Oriental-Artigo em periódico indexado (ALICE).

Kato, O. R., Kato, M. D. S., de Carvalho, C. J. R., Figueiredo, R. D. O., Camarão, A. P., \& Sá, T. D. A. (2007). Plantio direto na capoeira: uma alternativa com base no manejo de recursos naturais. Embrapa Amazônia Oriental-Capítulo em livro científico (ALICE)

Mackensen, J., Holscher, D., Kllnge, R. \& Folter, H., (1996). Nutrient transfer to the atmosphere by burning of debris in eastern Amazonia. Forest Ecology and Management, 86(1-3): 121 -128. DOI: https://doi.org/10.1016/S0378-1127(96)03790-5

Marcolan, A. L., Locatelli, M. \& Fernandes, S. R., (2009). Atributos químicos e físicos de um Latossolo e rendimento de milho em diferentes sistemas de manejo da capoeira: 1-6. Embrapa (Comunicado Técnico 352), Porto Velho - RO.

Mattos, L., Brondízio, E. S., Romeiro, A., \& Orair, R. (2010). Agricultura de pequena escala e suas implicações na transição agroecológica da Amazônia brasileira. Amazônica, 2 (2): 264-292. http://dx.doi.org/10.18542/amazonica.v2i2.399.

Melo, A. C. G. \& Durigan, G. (2010). Impacto do fogo e dinâmica da regeneração da comunidade vegetal em borda de Floresta Estacional Semidecidual. Revista brasileira de Botânica, 33 (1): 37-50. https://doi.org/10.1590/S0100-84042010000100005.

Ministério do Meio Ambiente. Agricultura Sustentável- MMA. (2000). https://www.mma.gov.br/informma/item/3045-governo-estimulaagriculturasustentavel-na-amazonia

Nair, P.K.R, (1993). An introduction to agroforestry. Kluwer Academic Press. The Netherlands. 449p.

Oliveira, J. S. R., Kato, O. R., Oliveira, T. F., Queiróz, J. \& Cardoso, R. (2007) Agricultura familiar e safs: produção com conservação na Amazônia Oriental, nordeste paraense. In: congresso brasileiro de sistemas de produção. Agricultura familiar, políticas públicas e inclusão social. 2007. Anais.Fortaleza: Embrapa Agroindústria Tropical.

Rego, A. K. C., \& Kato, O. R. (2018). Agricultura de corte e queima e alternativas agroecológicas na Amazônia. Novos Cadernos NAEA, Belém, 20 (3), ISSN 2179-7536. http://dx.doi.org/10.5801/ncn.v20i3.3482

Sá, T. D. A., Kato, O. R., Carvalho, C. J. R. \& Figueiredo, R. O., (2007). Queimar ou não queimar? De como produzir na Amazônia sem queimar. São Paulo: Revista USP: 72:90-97. https://doi.org/10.11606/issn.2316-9036.v0i72p90-97

Sampaio, C. A., Kato, O. R. \& Silva, D.N., (2008). Sistema de corte e trituração da capoeira sem queima como alternativa de uso da terra, rumo a sustentabilidade florestal no Nordeste paraense. Revista Gestão Social e Ambiental. 2:41-53, https://doi.org/10.24857/rgsa.v2i1

Santos, I. A., Brienza junior, S. \& Alegre, J., (2007). Agricultura sem fogo na Amazônia Oriental: formiga como indicador agroecológico: 53-56. Embrapa Amazônia Oriental (Artigo em periódico indexado), São Paulo.

Santos, J. C, (2008). Sustentabilidade socioeconômica e ambiental de sistemas de uso da terra da agricultura familiar no estado do Acre. 1-182. Tese (Doutorado em Economia Aplicada) - Universidade Federal de Viçosa, Viçosa.

Silva, J. M. N., Carreiras, J. M.B., Rosa, I. \& Pereira, J. M. C. (2011). Greenhouse gas emissions from shifting cultivation in the tropics, including uncertainty and sensitivity analysis. Journal of Geophysical Research, 116 (3): 1-21. https://doi.org/10.1029/2011JD016056.

Sollins, P., Spycher, G \& Gliessman, C.A, (1984). Net nitrogens mineration from lightand heavy-fraction forest soil organic matter. Soil Biology and Biochemistry 16 (1): 31-37. https://doi.org/10.1016/0038-0717(84)90122-6.

Sousa, S. G. A., Araujo, M. I., \& Wandelli, E. V. (2015). Saberes tradicionais dos povos amazônicos no Contexto do processo de transição agroecológica. Revista

AmbientalMente Sustentable (AMS). 2(20), 1696 - 1717. 10.17979/ams.2015.2.20.1694.

Thomaz, E. L. (2013). Slash-and-burn agriculture: Establishing scenarios of runoff and soil loss for a five-year cycle. Agriculture, Ecosystems and Environment, 168 (3). 10.1016/j.agee.2013.01.008. 